\title{
Hipoparatireoidismo primário em cão
}

\section{Primary hypoparathyroidism in a dog}

\author{
Mateus do Amaral Freitas ${ }^{1}$; Paulo César Jark ${ }^{1 *}$; Mariana Isa Poci Palumbo²; \\ Maria Lucia Gomes Lourenço ${ }^{3}$; Luiz Henrique de Araujo Machado ${ }^{3}$
}

\section{Resumo}

O hipoparatireoidismo é uma endocrinopatia raramente descrita em cães, provocada pela deficiência na síntese de paratormônio (PTH). A falta do PTH leva a hipocalcemia e hiperfosfatemia, resultando em uma série de alterações neurológicas e neuromusculares. Ao contrário da maioria das endocrinopatias, o hipoparatireoidismo é uma enfermidade em que a reposição hormonal exógena ainda não é viável, o que torna o tratamento um desafio. O presente relato tem como objetivo descrever um caso de hipoparatireoidismo primário em cão da raça Schnauzer com convulsões e alterações neuromusculares e o sucesso na terapia empregada, sendo este o primeiro caso, de acordo com a literatura consultada, de hipoparatiroidismo diagnosticado no Brasil. O hipoparatireoidismo deve ser considerado um diagnóstico diferencial para casos de convulsão. A avaliação neurológica completa e a dosagem dos níveis séricos do cálcio ionizado e do paratormônio são fundamentais para o diagnóstico desta doença. O diagnóstico precoce pode melhorar a qualidade de vida dos animais acometidos, pois após o início da terapia, há remissão completa dos sinais clínicos.

Palavras-chave: Deficiência, hipocalcemia, paratormônio, cão

\begin{abstract}
The hypoparathyroidism is a rare endocrinopathy reported in dogs, caused by a deficiency in the synthesis of parathyroid hormone (PTH). The lack of PTH causes hypocalcemia and hyperphosphatemia, resulting in a series of neurological and neuromuscular disorders. Unlike most endocrinopathies, hypoparathyroidism is a disease in which the exogenous hormone replacement is not being viable, becoming the treatment a challenge. The present report aims to describe a case of primary hypoparathyroidism in a Schnauzer dog with seizures and neuromuscular disorders, and successful treatment employed, this being the first case, according to the literature, of hypoparathyroidism diagnosed in Brazil. The hypoparathyroidism should be considered as a differential diagnosis in cases of seizure. A complete neurological evaluation and determination of serum ionized calcium and parathyroid hormone are essential for the diagnosis of this disease. Early diagnosis may improve the quality of life of affected animals, since after the initiation of therapy, there is complete remission of clinical signs.
\end{abstract}

Key words: Deficiency, hypocalcemia, parathyroid hormone, dog

\footnotetext{
${ }^{1}$ Médicos Veterinários Residentes, FMVZ/UNESP, Faculdade de Medicina Veterinária e Zootecnia, Universidade Estadual Paulista "Júlio Mesquita Filho", campus Botucatu, Botucatu, SP. E-mail: matias freitas@hotmail.com; paulocjark@hotmail.com

${ }^{2}$ Discente de Doutorado, FMVZ/UNESP, campus Botucatu, Botucatu, SP. E-mail: palumboma11@yahoo.com.br

${ }^{3}$ Profs. Drs. Assistentes, FMVZ/UNESP, campus Botucatu, Botucatu, SP. E-mail: mege@fmvz.unesp.br; henrique@fmvz.unesp. br

* Autor para correspondência
} 


\section{Introdução}

O hipoparatireoidismo primário é uma doença endócrina raramente descrita em cães (BURK; SCHAUBHUT, 1975; RUSSEL et al., 2006), resultante da deficiência absoluta ou relativa de paratormônio (PTH) (HIGGINS; ROSSMEISL, 2005). Tal deficiência causa várias anormalidades fisiológicas como hipocalcemia e hiperfosfatemia (MEYER; TERRELL, 1976; HENDERSON; MAHONY, 2005a).

Os principais sinais clínicos observados são: convulsão, tremores e fasciculações musculares, andar rígido, alterações comportamentais, febre, fraqueza, poliúria e polidipsia (BRUYETTE; FELDMAN, 1988; RUSSEL et al., 2006). A função da paratireoide pode ser avaliada de forma indireta pela mensuração de eletrólitos e, diretamente, pela quantificação da concentração do PTH (HENDERSON; POWERS; PERRY, 1991).

O cálcio é essencial para a liberação da acetilcolina durante a transmissão neuromuscular e para a estabilização da membrana das células nervosas (HIGGINS; ROSSMEISL, 2005). Quando sua concentração extracelular declina até níveis abaixo dos normais, o sistema nervoso torna-se progressivamente mais excitável devido ao aumento da permeabilidade da membrana neuronal (HIGGINS; ROSSMEISL, 2005). Esse aumento na excitabilidade ocorre tanto no sistema nervoso periférico quanto central, embora a maioria dos sinais clínicos manifeste-se perifericamente (HENDERSON; MAHONY, 2005a).

Ao contrário da maioria das endocrinopatias, o hipoparatireoidismo é uma enfermidade em que a reposição hormonal exógena ainda não é viável, o que torna o tratamento um desafio. O presente relato tem como objetivo descrever um caso de hipoparatireoidismo primário em cão e o sucesso na terapia empregada, sendo este o primeiro caso, de acordo com a literatura consultada, de hipoparatiroidismo relatado no Brasil.

\section{Relato do Caso}

Foi atendido no Serviço de Clínica Médica de Pequenos Animais do Hospital Veterinário da FMVZ-Unesp campus de Botucatu, um cão da raça Schnauzer, macho, não castrado, nove anos de idade com o histórico de crises convulsivas generalizadas há cinco dias. As crises convulsivas se caracterizavam por episódios focais com evolução para movimentos tônico-clonicos generalizados. Além das crises convulsivas, o paciente apresentava fricção facial, movimentos involuntários da orelha externa e dos membros pélvicos, fasciculações musculares, taquipneia, taquicardia, hipertermia e dor à palpação dos membros.

Não foram observadas alterações no hemograma e no exame de urina tipo 1. Nos exames bioquímicos (concentrações séricas de creatinina, ureia, cálcio, fósforo, sódio, potássio, magnésio, ALT, GGT, colesterol e triglicérides) foi encontrado concentração de fosfatase alcalina aumentada (400 UI/L, referência 20-156 UI/L). As concentrações séricas de cálcio corrigido $(2,5 \mathrm{mg} / \mathrm{dL}$ referência: 9,0-11,3 $\mathrm{mg} / \mathrm{dL})$ e cálcio ionizado $(0,33 \mathrm{mmoL} / \mathrm{L}$ referência 1,12-1,40 mmoL/L) estavam diminuídas e o fósforo discretamente aumentado $(6,47 \mathrm{mg} / \mathrm{dL}$ referência:0,5-6,2 mg/dL). O animal não apresentava histórico de medicações prévias que pudessem estar relacionadas a hipocalcemia, nem histórico de trauma cervical que pudesse comprometer a função da paratireoide.

Devido a suspeita de um distúrbio no metabolismo do cálcio, foi realizada a dosagem do paratormônio $(\mathrm{PTH})$, que estava diminuído $(0,20$ $\mathrm{pmoL} / \mathrm{L}$ referência $0,5-5,8 \mathrm{pmoL} / \mathrm{L})$. A análise dos sinais clínicos, associados ao histórico do paciente e aos exames laboratoriais, confirmou o diagnóstico de hipoparatireoidismo primário.

Instituiu-se o tratamento ambulatorial com administração intravenosa in bolus lento de $10 \mathrm{~mL}$ de gluconato de cálcio $10 \%$ diluídos em $15 \mathrm{~mL}$ de solução fisiológica, associada a infusão contínua de gluconato de cálcio a $10 \%$, diluído em $250 \mathrm{~mL}$ de 
solução fisiológica na taxa de infusão de $1,5 \mathrm{~mL} /$ $\mathrm{kg} /$ hora durante sete dias. Após este período, instituiu-se o tratamento de manutenção, através da administração de carbonato de cálcio a cada 12 horas $(50 \mathrm{mg} / \mathrm{kg})$, administrado junto com a alimentação e $0,005 \mathrm{mcg} / \mathrm{kg}$ de calcitriol por via oral, 20 minutos após a alimentação.

O animal apresentou melhora clínica progressiva logo após a infusão de cálcio com remissão dos sinais clínicos, porém apresentava episódios de hipocalcemia com retorno das manifestações clínicas. Após oito dias do início da terapia intravenosa o quadro se estabilizou e o animal não apresentou mais episódios de hipocalcemia. Foram realizados exames laboratoriais semanais para avaliação das concentrações séricas do cálcio e fósforo e o tratamento foi ajustado de acordo com os resultados destes exames. A melhora dos sinais clínicos e estabilização do paciente foram evidentes quando o animal apresentou concentração sérica de cálcio superior a $8 \mathrm{mg} / \mathrm{dL}$. A partir dessa concentração, suspendeu-se a administração intravenosa de gluconato de cálcio. Durante o tratamento, houve aumento dos níveis séricos do cálcio, juntamente com o aumento da concentração sérica do fósforo, sendo necessário ajuste nutricional associado a prescrição de hidróxido de alumínio (uma cápsula de $300 \mathrm{mg}$ a cada 24 horas) como quelante de fósforo, a fim de manter os níveis séricos de 11-13 mg/dL de cálcio e 5-7 mg/dL de fósforo. O tratamento oral foi mantido e 17 meses após seu início, o animal encontra-se estável e as monitorações mensais dos níveis séricos de cálcio e fósforo estão dentro da normalidade.

O hipoparatireoidismo primário geralmente acomete cães de meia idade (média de quatro a oito anos) sendo mais comum, aproximadamente 65\% dos casos, em fêmeas (HENDERSON; MAHONY, 2005a). O paciente era macho e encontravase, no momento do diagnóstico, acima da faixa etária descrita nos casos de hipoparatireoidismo. As raças mais comumente acometidas por hipoparatireoidismo são os Poodles, Schnauzers miniatura, Pastores Alemães, Labradores e raças Terriers (FELDMAN, 2010). Em um trabalho que avaliou 17 casos de hipoparatireoidismo canino, a raça mais acometida foi o São Bernardo e cães sem raça definida (RUSSEL et al., 2006).

As duas principais causas de hipoparatireoidismo em cães são a atrofia ou destruição idiopática da paratireóide e lesão secundária a trauma cervical ou remissão iatrogênica durante cirurgia na tireoide (PETERSON, 1982). Devido ao fato do animal não ter sido submetido a procedimento cirúrgico e não ter histórico de trauma na região, a causa mais provável neste caso seria a atrofia ou destruição idiopática da paratireoide. Os principais diagnósticos diferenciais dos quadros de hipocalcemia em machos incluem hipoproteinemia, hipomagnesemia, pancreatite, administração de certos medicamentos, má absorção intestinal, intoxicação por etilenoglicol e insuficiência renal (PETERSON, 1982; RUSSEL et al., 2006), distúrbios que foram excluídos com a avaliação do histórico do animal, associado aos resultados dos exames laboratoriais realizados. O diagnóstico definitivo de hipoparatiroidismo primário foi realizado com base no histórico, sinais clínicos associados a hipocalcemia e concentração sérica diminuída de PTH. O aumento da concentração de fosfatase alcalina apresentado pelo animal pode estar relacionado à colestase hepática prévia, pois o animal não apresentava histórico ou sinais clínicos compatíveis com outras causas de aumento de fosfatase alcalina como administração de medicamentos, doenças neoplásicas ósseas, doenças endócrinas como o hiperadrenocorticismo (FELDMAN, 2010).

As convulsões secundárias ao hipoparatireoidismo podem ser generalizadas ou focais e ocorrem em $80 \%$ dos cães com esta endocrinopatia (PETERSON, 1982; HENDERSON; MAHONY, 2005a; FELDMAN, 2010). Outros sinais descritos pela literatura como fricção facial intensa, mordedura e lambedura das patas e períodos de ofego (HENDERSON; MAHONY, 2005a; RUSSEL et al., 2006; FELDMAN, 2010), foram observados 
neste caso. A fricção facial intensa provavelmente ocorre em decorrência da dor associada às cãibras dos músculos masseter e temporal e/ou ainda pela sensação de "formigamento" ao redor da boca nos cães (HENDERSON; MAHONY, 2005a; FELDMAN, 2010).

A gravidade dos sinais clínicos está relacionada a duração e magnitude da diminuição dos níveis séricos de cálcio e com outras alterações eletrolíticas associadas (HENDERSON; MAHONY, 2005a). Os sinais tendem também a se manifestar de modo intermitente como descrito no presente caso, intercalados com períodos relativamente normais que duram minutos a dias e tendem a se agravar com o exercício (RUSSEL et al., 2006). Mesmo nos episódios sem sinais clínicos, os animais permanecem hipocalcêmicos, porém, o mecanismo exato dessa manifestação não está totalmente elucidado (HENDERSON; MAHONY, 2005a; FELDMAN, 2010).

A suplementação de cálcio é um importante componente para o tratamento do hipoparatireoidismo e deve ser instituído o mais rápido possível, pois os sinais clínicos são reversíveis após o início da terapia (HIGGINS; ROSSMEISL, 2005). A suplementação oral de cálcio pode ser fornecida na forma de diferentes sais como gluconato, lactato, cloreto ou carbonato (RUSSEL et al., 2006; FELDMAN, 2010). A vitamina D é administrada para aumentar a absorção de cálcio pelo trato intestinal (FELDMAN, 2010). A administração de calcitriol na dosagem inicial de 0,005-0,01 micrograma $/ \mathrm{kg} /$ dia junto com os suplementos orais de cálcio é fundamental no início da terapia de manutenção. O calcitriol (1,25-dihidroxivitamina $\mathrm{D}_{3}$ ) é o fármaco de escolha para o tratamento de manutenção do hipoparatireoidismo por oferecer vantagens como início de ação mais rápido e menor meia-vida (dois a quatro dias), o que diminui os riscos de toxicidade se ocorrer o desenvolvimento de hipercalcemia durante o tratamento (RUSSEL et al., 2006).
O carbonato de cálcio tem sido utilizado como primeira opção para o tratamento do hipoparatireoidismo em medicina, por ter alta concentração de cálcio (40\%), ser amplamente disponível, ter baixo custo e não apresentar efeitos colaterais no sistema gastrointestinal (HENDERSON; MAHONY, 2005b). Embora não haja pesquisas em medicina veterinária recomendando qual sal deva ser utilizado, no cão deste relato, optou-se pelo carbonato de cálcio pelas razões descritas acima. A dose oral do cálcio deve ser reajustada conforme a concentração sérica de cálcio (HENDERSON; MAHONY, 2005b). A terapia instituída para o animal deste caso resultou na remissão dos sinais clínicos.

Segundo a literatura consultada, este é o primeiro relato de hipoparatireoidismo em cão realizado no Brasil. O hipoparatireoidismo deve ser considerado um diagnóstico diferencial para casos de convulsão. A avaliação neurológica completa e a dosagem dos níveis séricos do cálcio ionizado e do paratormônio são fundamentais para o diagnóstico desta doença. O diagnóstico precoce pode melhorar a qualidade de vida dos animais acometidos, pois após o início da terapia, há remissão completa dos sinais clínicos.

\section{Referências}

BRUYETTE, D. S.; FELDMAN, E. C. Primary hypoparathyroidism in the dog. Report of 15 cases and review of 13 previously reported cases. Journal of Veterinary Internal Medicine, United Kingdom, v. 2, n. 1, p. 7-14, 1988.

BURK, R. L.; SCHAUBHUT, C. W. Spontaneous primary hypoparathyroidism in a dog. Journal of the American Animal Hospital Association, United States of America, v. 11, n. 6, p. 784-785, 1975.

FELDMAN, E. C. Disorders of the parathyroid glands. In: ETTINGER, S. J.; FELDMAN, E. C. (Ed.). Textbook of veterinary internal medicine. Philadelphia: WB Saunders, 2010. p. 1722-1750.

HENDERSON, A. K.; MAHONY, O. Hypoparathyroidism: pathophysiology and diagnosis. Compendium, United States of America, v. 27, n. 4, p. 270-279, 2005a. 
Hypoparathyroidism: treatment. Compendium, United States of America, v. 27, n. 4, p. 280-287, 2005 b.

HENDERSON, R. A.; POWERS, R. D.; PERRY, L. Development of hypoparathyroidism after excision of laryngeal rhabdomyosarcoma in a dog. Journal of the American Animal Hospital Association, United States of America, v. 198, n. 4, p. 639-643, 1991.

HIGGINS, M. A.; ROSSMEISL, J. H. What is your neurologic diagnosis? Journal of the American Animal Hospital Association, United States of America, v. 226, n. 5, p. 699-701, 2005.
MEYER, D. J.; TERRELL, T. G. Idiopathic hypoparathyroidism in a dog. Journal of the American Animal Hospital Association, United States of America, v. 168 , n. 9, p. 858-860, 1976.

PETERSON, M. E. Treatment of canine and feline hypoparathyroidism. Journal of the American Animal Hospital Association, United States of America, v. 181, n. 11, p. 1434-1436, 1982.

RUSSEL, N. J.; BOND, K. A.; ROBERTSON, I. D.; PARRY, B. W.; IRWIN, P. J. Primary hypoparathyroidism in dogs: a retrospective study of 17 cases. Australian Veterinary Journal, Australia, v. 84, n. 8, p. 285-290, 2006. 
